\title{
Cinco años de crisis en el mercado de la comunicación (2008-2013)
}

\author{
Francisco CABEZUelo-LoRenzo \\ Universidad de Valladolid \\ cabezuelo@hmca.uva.es
}

\section{Resumen}

Este artículo resume los principales problemas de las empresas de medios de comunicación en el actual contexto de crisis económica que vive España, desde 2008 hasta la actualidad. Pone el acento en el caso de la industria de la publicidad. Hace una descripción del actual panorama mediático español marcado por un profundo descenso de la inversión publicitaria en medios tradicionales. La investigación demuestra que la crisis publicitaria ha forzado a los medios a cambiar su propia estructura como empresas.

Palabras clave: Empresas de comunicación, crisis económica, prensa, España.

\section{Five years of crisis the communication market (2008-2013)}

\begin{abstract}
This article tells the main problems of the mass media companies in the current state of economic crisis in Spain, from 2008 until now. It pays special attention to the case of the advertising industry. This work shows the current Spanish media landscape, determinate by the deep crisis and a low invest in traditional advertising. It explains how the advertising crisis has forced the media to change their own structures as corporations.
\end{abstract}

Keywords: Media companies, economic crisis, press, Spain.

Referencia normalizada:

Cabezuelo Lorenzo, F. (2013) Cinco años de crisis en el mercado de la comunicación (2008-2013). Historia y Comunicación Social. Vol. 18. № Especial Diciembre. Págs. 703-715.

715 .

Sumario: 1.- Introducción y contexto de la actual crisis. 2.- La crisis publicitaria: el caso de España. 3.- Las agencias de publicidad ante la crisis. 4.- La crisis en los medios de comunicación españoles. 5.- Conclusiones. 6.- Bibliografía y fuentes.

\section{Introducción y contexto de la actual crisis}

En la actualidad, nuestro país, al igual que otros tanto países de la Unión Europea, sufre una gran crisis económica y financiera que está creando serios problemas sociales. Algunos expertos como Álvaro Nadal (2008) ya corroboraron en su momento que el germen de esta crisis estadounidense al estallido de la denominada "burbuja punto.com", que tuvo lugar en el año 2001, a raíz de la cuál la Reserva Federal Americana aplicó una rebaja histórica en los tipos de interés, haciéndolos variar del 
6,5 al 1 por ciento, un cambio, sin duda, sin precedentes. Para otros muchos analistas, la actual crisis que estamos sufriendo todavía en algunos pasíes como España tiene su origen a mediados de 2007 en Estados Unidos. El mercado norteamericano de las hipotecas 'basura' o subprime, basado en la concesión de créditos a familias sin suficiente solvencia y dispuestas a hacer frente a elevados tipos de interés (Benítez y Cabrera, 2010), se configura como el principal factor causante de esta recesión a nivel mundial. En el caso concreto de nuesro país, la crisis fue fruto de la explosión de otros problemas, desde el año 2008 en adelante, como el final de la burbuja inmobiliaria, la crisis bancaria de $2011 \mathrm{y}$, finalmente, el aumento del desempleo en España. Esta coyuntura se ha convertido para muchos en el germen de nuevos movimientos sociales (como sería el caso del llamado movimiento 15-M, Stop Desahucios o Indignados, entre otros) encaminados a cambiar el modelo económico y productivo así como cuestionar el sistema político y las actuales instituciones, exigiendo una renovación democrática y el fin de algunos privilegios políticos (Sotelo González, 2011). La industria de la comunicación y de la publicidad no es ajena a este entorno. El mundo de los negocios y por tanto de la publicidad también es susceptible, como elemento que es de todo este sistema, de sufrir las consecuencias del difícil entorno económico-financiero. Los mercados de la comunicación, y más concretamente el de la publicidad, no son ajenos a la coyuntura de recesión que actualmente afecta a la estructura productiva y de consumo de muchos países del mundo, pero que está especialmente afectando muy duramente a algunos países europeos, como es el caso de España.

\section{La crisis publicitaria: el caso de españa}

La crisis se extiende desde el año 2008 hasta la actualidad. Sin embargo, hace ahora justamente más de cuatro años que aparecieron algunas de las primeras noticias sueltas alentadoras sobre el futuro próximo de los medios. Así se han visto notas sobre la notable mejoría en la industria audiovisual china que veía cómo sus ingresos del año 2009 sumaban 28.700 millones de dólares, un 17,5\% más que en el ejercicio anterior (El Mundo, 19.05.2010). Sin embargo, las diferentes aportaciones del Gobierno a algunos medios, en forma de ayudas, desvirtúan, en cierta medida, la relevancia del dato. En el primer trimestre de 2010 la inversión publicitaria española cayó un 2,6 \%, una cifra considerablemente escasa si se compara con las de años anteriores: la inversión real estimada alcanzó en 2009 la cifra de 12.699,4 millones de euros, lo que supuso un descenso del 14,9 \% de este indicador en relación a los datos registrados en 2008, con 14.915,7 millones de inversión (InfoAdex 2010). Pero eso fue sólo el inicio de la crisis de la publicidad porque durante el pasado año 2012, la inversión publicitaria en España en medios se desplomó un 9,9\% en relación con el año anterior (2011), hasta alcanzar los 10.858,8 millones de euros, según el estudio anual de InfoAdex (2012). 
Según el informe de InfoAdex del ejercicio del año 2012, la principal caída tuvo lugar en los medios convencionales (televisión, Internet, radio, prensa) donde la inversión pasó de 5.497,1 millones de euros en 2011 a 4.630 millones de euros en el pasado año 2012, lo que supuso un descenso del 15,8\%. De este modo, en 2012 se produjo una contracción del mercado, tal y como ha ocurrido en los últimos cuatro años, con la excepción del año 2010 donde sí hubo un repunte positivo. Estos medios tradicionales o convencionales representaron el $42,6 \%$ de la inversión total producida en España en 2012. Por su parte, los denominados medios no convencionales como el mailing, márketing móvil, o el patrocinio deportivos contó con una inversión de 6.228 millones de euros durante 2012, lo que supone una caída del $5 \%$ respecto a 2011. Este segmento supuso el $57,4 \%$ de la inversión total de 2012, según la información tomada de la noticia publicada el 28 de febrero de 2013 por el diario catalán La Vanguardia.

Parece ser que Internet es el único soporte que resiste los embates de la crisis (Sotelo González y Salgado Santamaría, 2011). De hecho, la Red se ha convertido en España en el segundo medio por volumen de inversión dentro de medios convencionales. Según el informe de InfoAdex de 2012, se estima que esta tendencia continúe durante el presente año 2013, tal y como sucede en otros mercados de la Unión Europea. En otros países europeos, como es el caso de Reino Unido, Internet está en la primera posición. En medios no convencionales, el que mayor porcentaje alcanza es el mailing personalizado frente a otras fórmulas comerciales, lo que supuso un $30,2 \%$ del total de los medios no convencionales. En 2012 el sector de los medios no convencionales recibió una inversión de 1.881,6 millones de euros frente a los 1.914,1 millones recibidos en el año anterior, habiendo experimentado un decremento interanual del 1,7\%. A pesar de todo, sufrió el año pasado un descenso en inversión del 2,1\% en comparación con 2011, hasta los 880,5 millones de euros, lo que supone una cuota del 19\% sobre el total de la inversión en los medios convencionales.

Pero, sin duda alguna, la gran caída general la ha sufrido la televisión. De hecho, en 2012, según el informe de Infoadex, experimentó una caída de la inversión del 18,9\% durante el año pasado, con 1.815,3 millones de euros, y su cuota sobre la inversión total en medios convencionales se situó en el 39,2\% en 2012. En este apartado, destacan las televisiones nacionales en abierto, con 1.639 millones de euros, lo que supone una caída del 16,8\%. Por su parte, las televisiones autonómicas se quedaron en 2012 en una cantidad de 126,8 millones, equivalente a una caída del 36\% respecto a 2011. Respecto a los canales de televisión de pago el total invertido durante el pasado año fue de 43,1 millones, una cantidad es un $28,4 \%$ inferior a la de 2011 , mientras que en las televisiones locales el decrecimiento registrado en 2012 fue del $25 \%$, lo que sitúa la cifra obtenida de inversión en el año en 1,5 millones de euros.

Los diarios alcanzaron en el ejercicio 2012 un volumen de inversión publicitaria de 766,3 millones de euros (-20,8\%), situándose en el tercer lugar por su peso, representando el $16,5 \%$ del total de la inversión publicitaria recogida en los medios convencionales. La radio recibe en 2012 una inversión de 453,5 millones de euros (-13,6\% respecto a 2011) y el cine decrece un $12,7 \%$, hasta los 25,8 millones de euros. 
Respecto a los grandes anunciantes de 2012, según el citado informe de Infoadex del ejercio del año 2012, el primero fue Procter \& Gamble con 105,1 millones de euros invertidos durante el pasado año, lo que supone un $2,7 \%$ de la inversión total en medios convencionales en España. Infoadex registró en 2013 un total de 31.728 anunciantes el año pasado, si bien sólo 74 de ellos invirtieron por encima de 10 millones de euros y el $80,3 \%$ del total se acumula en 786 anunciantes. Si hablamos de productos y sectores, el mercados del automóvil, las finanzas, servicios de distribución, belleza e higiene y alimentación supusieron el $48 \%$ de la inversión publicitaria en medios convencionales, cerca de los 1.800 millones de euros entre los cinco.

Estos datos permiten afirmar que la disminución ha repercutido en mayor medida en España en los medios convencionales que en los no convencionales, siendo Internet el único medio convencional que no ha registrado una caída en este indicador, símbolo del auge que están experimentando los medios digitales (Tapia Frade, A. et alt, 2010).

\section{Las agencias de publicidad ante la crisis.}

Si acabamos de ver anteriormente en unos párrafos previos la inversión publicitaria en nuestro país y su influencia en los medios de comunicación, como anunciantes, ahora corresponde hacernos la pregunta desde nuestro campo, desde la Publicidad. ¿Quiénes han sido los que se han comido esa parte del pastel? Contamos con los datos de estudio de InfoAdex de Agencias de Publicidad y Agencias de Medios 2012, que hacer referencia a los datos de 2011 pero todavía no contamos con los datos del pasado año 2012. El último informe es número 13 de InfoAdex.

Estudio cuenta con el análisis de las cuentas de 44 agencias de publicidad que han colaborado en él y que han gestionado en su conjunto durante 2011 un total de 2.574 marcas directas con inversión controlada, pertenecientes a un total de 1.030 anunciantes. La inversión atribuida a las agencias presentes en el estudio asciende a 2.588 millones de euros, cifra que es el 54,9\% del total de la inversión publicitaria controlada por InfoAdex en valores estimados como reales en el año 2011.

Esa decimotercera edición de su análisis publicada en el pasado 2012 nos sirve para este trabajo y para realizar un análisis global. El trabajo de InfoAdex, que cada año observa a los principales sujetos del sector publicitario, cuenta con un estudio propio para las agencias de publicidad y las agencias de medios, con los anunciantes y marcas para las que trabajan.

El estudio del verano de 2012 analiza la inversión publicitaria gestionada por las agencias, entendiendo como tal la repercusión económica de las campañas y acciones publicitarias hechas por las agencias en los medios convencionales, en términos de la inversión controlada por esta firma en valores tanto estimados como reales. En el siguiente cuadro aparecen enumeradas las treinta primeras agencias en razón de su 
volumen de inversión gestionada en el año 2011 según los datos hechos públicos el pasado verano de 2012:

\begin{tabular}{|c|c|c|}
\hline & Agencias de Publicidad & Inversión controlada gestionada(€) \\
\hline 1 & MCCANN & 263.470 .534 \\
\hline 2 & BASSAT, OGILVY \& MATHER & 251.848 .940 \\
\hline 3 & GREY & 233.336 .032 \\
\hline 4 & EURO RSCG ESPAÑA & 221.300 .060 \\
\hline 5 & YOUNG \& RUBICAM & 194.104 .993 \\
\hline 6 & DDB & 183.684 .890 \\
\hline 7 & SRA. RUSHMORE & 168.233 .778 \\
\hline 8 & TBWA & 124.255 .540 \\
\hline 9 & PUBLICIS ESPAÑA & 123.438 .827 \\
\hline 10 & LEO BURNETT & 115.766 .368 \\
\hline 11 & CONTRAPUNTO BBDO & 112.624 .593 \\
\hline 12 & SCPF & 106.878 .477 \\
\hline 13 & JWT DELVICO & 81.128 .117 \\
\hline 14 & SAATCHI \& SAATCHI & 68.574 .547 \\
\hline 15 & ZAPPING M\&C SAATCHI PUBLICIDAD & 47.319 .721 \\
\hline 16 & SHACKLETON & 46.521 .507 \\
\hline 17 & TIEMPO BBDO & 34.171 .634 \\
\hline 18 & GRUPO NOSTRUM RNL & 27.883 .670 \\
\hline 19 & VILLARROSAS & 27.570 .899 \\
\hline 20 & IGRIEGA & 25.498 .191 \\
\hline 21 & ROAD & 18.384 .327 \\
\hline 22 & PAVLOV & 16.955 .984 \\
\hline 23 & EUREKA/N.W.AYER & 15.344 .132 \\
\hline 24 & BAP\&CONDE & 14.131 .931 \\
\hline 25 & DARWIN \& CO & 13.780 .820 \\
\hline 26 & NEXT DOOR & 11.054 .228 \\
\hline 27 & COMPACT FMRG & 10.621 .081 \\
\hline 28 & RICARDO PEREZ ASOCIADOS & 5.609 .063 \\
\hline 29 & SINTESIS SPC & 5.377 .931 \\
\hline 30 & KASTNER \& PARTNERS & 4.578 .645 \\
\hline
\end{tabular}

Tabla tomada del informe de InfoAdex 2012 publicada por www.marketingdirecto.com

Este mismo informe del pasado verano 2012 también cubre las agencias de medios y su actividad medida en 2011. En cuanto a las agencias de medios, InfoAdex analizó y publicó las cuentas de 24 agencias de medios que colaboraron en el estudio. Se 
habla de una cobertura que asciende a 4.947 marcas directas pertenecientes a 2.116 anunciantes. Estas cifras corresponden a una inversión gestionada atribuida a las agencias de medios asciende a 3.388,1 millones de euros lo que representa el 71,8\% de la inversión publicitaria controlada por InfoAdex en valores estimados como reales en el año 2011, según el informe del pasado verano de 2012:

\begin{tabular}{|c|c|c|}
\hline \multicolumn{2}{|r|}{ Agencias de medios } & Inversión controlada gestionada en $2011(\epsilon)$ \\
\hline 1 & MPG & 679.091 .943 \\
\hline 2 & OPTIMEDIA & 358.276 .412 \\
\hline 3 & OMD & 315.196 .560 \\
\hline 4 & ZENITH & 262.104 .908 \\
\hline 5 & ARENA MEDIA COMUNICATIONS & 259.468 .542 \\
\hline 6 & MEDIACOM IBERIA & 225.196 .231 \\
\hline 7 & $\begin{array}{l}\text { MEC (total compra) } \\
\text { - FOCUS MEDIA }\end{array}$ & 203.629 .261 \\
\hline 8 & MINDSHARE & 198.583 .361 \\
\hline 9 & STARCOM & 184.987 .030 \\
\hline 10 & YMEDIA & 137.549 .580 \\
\hline 11 & EQUMEDIA XL & 115.389 .745 \\
\hline 12 & MAXUS & 105.013 .894 \\
\hline 13 & UNIVERSAL MCCANN & 91.821 .377 \\
\hline 14 & INITIATIVE & 70.257 .007 \\
\hline 15 & VERITAS & 60.953 .495 \\
\hline 16 & PHD & 23.014 .688 \\
\hline 17 & ALMA MEDIA & 22.932 .656 \\
\hline 18 & DATAPLANNING & 18.898 .021 \\
\hline 19 & NEGOCIACION Y COMPRA ADVERTISING & 13.533 .452 \\
\hline 20 & OUTDOORMEDIA (solo exterior) & 12.544 .567 \\
\hline 21 & MEDIA BY DESIGN SPAIN & 12.327 .921 \\
\hline 22 & ORANGE MEDIA ADV.,S.L. & 10.118 .090 \\
\hline 23 & EMV.DOS & 7.199 .113 \\
\hline & Total general & 3.388 .087 .854 \\
\hline
\end{tabular}

Tabla tomada del informe de InfoAdex 2012 publicada por www.marketingdirecto.com

Respecto a los datos de inversión publicitaria según las agencias de publicidad, hay que aclarar que el estudio cubre la inversión en medios convencionales (televisión, incluyendo tanto a las cadenas nacionales y autonómicas que emiten en abierto como a las televisiones de pago, diarios, revistas, radio, dominicales, cine, publicidad exterior e Internet) y no se incluyen ni la inversión en medios no convencionales ni la correspondiente a investigación, producción, honorarios de agencias, ni tampoco otros conceptos de inversión relacionados con la publicidad o comunicación comercial. 
Lógicamente, la inversión viene siempre referida al ámbito nacional, no computándose la que se lleva a cabo en medios internacionales. La base fundamental del estudio son las cifras de inversión publicitaria controladas por InfoAdex, valoradas con la metodología y criterios empleados en la elaboración del Estudio InfoAdex de la Inversión Publicitaria en España 2012, que maneja los datos publicitarios del 2011.

Los nombres de los anunciantes y de las marcas para las que han trabajado en 2011 las agencias de publicidad y las agencias de medios que aparecen en el estudio proceden de las propias empresas, que han proporcionado dicha información respondiendo a los cuestionarios enviados. Las inversiones gestionadas que aparecen en el estudio se obtienen al atribuir desde InfoAdex a cada una de estas cuentas declaradas la inversión controlada y estimada como real según el Estudio InfoAdex de la Inversión Publicitaria en España 2012.

\section{La crisis en los medios de comunicación españoles}

Mercado publicitario y medios de comunicación están estrechamente ligados. Mientras el año pasado año la inversión publicitaria repuntó ligeramente, después de un descenso sostenido, en medios como radio y televisión, con un crecimiento interanual de un $2 \%$ y un $4 \%$ respectivamente, según InfoAdex, pero cayó en revistas $(-1 \%)$ y en diarios $(-4,2 \%)$. Como ya hemos dicho, en los últimos 24 meses, sin embargo, las malas noticias se han repartido en todos los medios de comunicación, excepto Internet, y los estudios sitúan la caída de la inversión publicitaria del primer semestre del año en cerca de un $6 \%$. Pero esta misma medida ha caído en prensa diaria entre un $11 \%$ y un $12 \%$, según la consultora i2p o InfoaAdex, y las previsiones reconocidas por los editores de diarios rondan una caída en todo el año del $9 \%$,aunque el descenso será menor en el caso de los dominicales, según la FAPE.

Los medios de comunicación han pagado muy cara la crisis y el descenso de la inversión publicitaria. La televisión privada está separada en dos grandes bloques Mediaset España (con Telecinco y Cuatro) y Atresmedia (Antena 3 y La Sexta TV). Y luego por otro lado están las televisiones públicas con sus terribles pérdidas económicas. En 2012, las dos grandes televisiones privadas de nuestro país, Antena 3 TV y Telecinco vieron reducidos sus beneficios en más de un $60 \%$, en plena debacle de la publicidad. Según las estimaciones realizadas por distintos servicios de estudios, las ganancias conjuntas de ambos grupos rondaron los 80 millones de euros, muy lejos de los casi 204 millones que sumaron en el pasado ejercicio. Eso sí, al menos no están en números rojos a pesar de soportar un descenso de la publicidad del $20 \%$ en el último trimestre del año 2012.

En el caso de Antena 3 TV (Atresmedia) la consultora, JB Capital Markets adelantó un beneficio neto de 23,1 millones de euros para este canal, lo que supone un descenso del 75,3\% en relación a los 93,4 millones de 2011. La cadena presidida por el empresario José Manuel Lara, aunque contuvo la caída de la publicidad y sólo 
registró un descenso del 8,2\% -perdió unos 65 millones-, incrementó en 2012 considerablemente los gastos, que pasaron de 680,8 a 704 millones. La comparación en costes es dura respecto al año anterior debido a la inclusión de la Fórmula 1 y nuevos programa en prime time. El beneficio bruto operativo se situaría en 33,2 millones, un $73,3 \%$ menos, según la noticia publicada por El Confidencial en su momento.

Según la noticia que recoge el informe de la consultora JP Morgan calca prácticamente las cifras de facturación y gastos y vaticina un beneficio neto de 22 millones de euros. No prevé una mejoría significativa en términos publicitarios para 2013, año para el que concede a la firma de Silvio González una previsión de beneficio de 27 millones. Todo pese a que España es, junto al Reino Unido, el mercado más atractivo de Europa para invertir en medios. Los mercados parecen ahora más proclives a invertir en la periferia, según este informe. En el caso de España, el número de cadenas de televisión en abierto se ha reducido de seis en 2009 a tres en 2012, con dos operadores controlando el $90 \%$ del mercado publicitario, que son Mediaset España y Atresmedia.

Las estimaciones de JB Capital Markets para Mediaset duplican el beneficio neto de Antena 3. La cadena de Paolo Vasile alcanza unas ganancias de 44,9 millones, pese a sufrir una caída de la facturación publicitaria del 14,5\% y perder el umbral de los 1.000 millones de ingresos. La compañía logra unas ventas por anuncios de 860,5 millones, a los que añade un importante empujón de fondos llovidos del cielo por la explotación de la exitosa película 'Lo imposible'. Los costes, contenidos, apenas crecen un 3\%, hasta los 841 millones. JP Morgan, sin embargo, incrementa la posible facturación de la firma -hasta los 881 millones- y prevé beneficios de 59 millones de euros, lejos en todo caso de los 110 del ejercicio anterior.

La situación de Mediaset España y de Atresmedia es diferente. Mediaset ha sido tradicionalmente una máquina de hacer dinero cuyos beneficios netos han alcanzado la cifra de los 2.000 millones en la última década, Sin embargo, a mediados de 2012 Mediaset España anunció su intención de destinar al pago de dividendo un 50\% de su beneficio neto de 2011, después de haber mantenido desde 2004 una retribución a su accionista superior al $90 \%$ de sus ganancias. Según la noticia publicada por El Mundo, esta medida suponía destinar la nada despreciable cantidad de 55,25 millones a remunerar al accionariado. Con un saldo positivo de 2012 que no llegó a los 60 millones de euros, lo razonable es mantener esa política conservadora.

Bien diferente es la situación de Atresmedia, cuyo principal accionista, la editorial Planeta, de José Manuel Lara, necesita del dividendo para cumplir con sus vencimientos con la banca. Ya quedó claro en diciembre pasado, cuando el grupo acordó distribuir a cuenta de los resultados del año 2012 la cantidad de 0,11 euros por acción. Una entrega suponía a la compañía un pago el 20 de diciembre de 21,35 millones de euros y anticipaba un abono similar a mediados de 2013, según los informes de las consultoras reproducidos por la prensa general y especializada. No en vano, Antena 3 abona tradicionalmente un $50 \%$ del dividendo total a cuenta en la última parte 
del ejercicio y desembolsa otra parte, complementaria, hacia el mes de junio, según informaciones publicadas por El Mundo.

Las cifras para este año 2013 están siendo muy duras respeto a la inversión publicitaria. La cadena Antena 3 TV ha sido la primera en salir a la palestra y mostrar sus desalentadoras cifras, ya que la cadena de José Manuel Lara confesaba un desplome de la facturación del $16 \%$ entre enero y marzo de 2013, lo que apenas le reportaba un beneficio de un millón de euros. De este modo, inmediatamente, los gestores del grupo anunciaban una revisión de la hoja de ruta publicitaria para este año 2013, asumiendo una caída de la facturación del $15 \%$ frente al $10 \%$ contemplado hasta ahora. Para los analistas de El Confidencial, "no será el último medio en escenificar sus estrecheces" porque consideran que se será difícil obtener dinero para una cadena "con costes fijos de programación muy elevados y difíciles de recortar, que el año pasado ya flirteó puntualmente con los números rojos".

Además, los datos de InfoAdex publicados por El Confidencial sobre la inversión publicitaria en España en el primer trimestre de 2013, viene a decirnos que está sólo alcanzó los 828 millones de euros, un 16,6\% menos que el año anterior, cuando entre enero y marzo se alcanzaban los 993,5 millones, tan sólo un año antes. Para algunos analistas, este enfoque es aún más preocupante si se amplía la muestra, dado que los medios convencionales se repartieron 7.983,6 millones en el año 2007, fecha de incio de la crisis, según el análisis de los expertos de El Confidencial.

Si el pasado año 2012 se cerró con unos beneficios de 4.630 millones y este año amenaza con dejar la recaudación en la mitad en apenas un lustro. Las expectativas de repunte en la segunda parte del ejercicio empiezan a esfumarse ya que la publicidad no es ajena a la crisis económica, al paro, a la falta de inversión y los recortes o medidas de ahorro de administraciones públicas y empresas privadas.

Los datos pueden ser todavía más duros para las televisiones privadas agrupadas en UTECA si vuelve la publicidad a la corporación RTVE, ya que la situación se puede agrabar si el actual Gobierno de España, apoyado por la mayoría del Partido Popular, cumple finalmente con su proyecto de devoler los anuncios para mantener RTVE en caso de que la Comisión Europea tumbe desde Bruselas el actual modelo de financiación. De acuerdo con algunos analistas, la vuelta de RTVE al reparto del pastel publicitario restaría al sector en torno a 100 millones de euros, lo que supondría un cálculo dramático tanto para el grupo de Antena 3 como para Mediaset. Tampoco serían buenas noticias para RTVE, que dejaría de recibir ingresos de las operadoras de telecomunicaciones, de las que está obteniendo cifras mayores. Dejar de recibir estos ingresos se condiera desde RTVE insuficiente para compensar lo que ahora recibe la televisión pública por esta polémica vía. Por su parte, en sede parlamentaria, el presidente de la cadena pública, Leopoldo González-Echenique, puesto en el cargo por el Gobierno del Partido Popular, se adelantaba a la críticas de la UTECA y se ponía la venda antes de la herida. González-Echenique argumentaba que la crisis también ha afectado a las empresas de telecomunicaciones, por lo que la corporación 
de RTVE sólo espera recaudar 120 millones a través de las operadoras de telefonía y contenidos digitales.

Sin duda alguna, el futuro de las televisiones españolas no es muy prometedor. El pastel publicitario cada día es más pequeño. Además, la postura de los medios de comunicación, ofreciendo poca calidad y productos sin interés no ayudará a salir de esta crisis. Uno de los ragos actuales de los medios de comunicación es la tendencia hacia contenidos más de entretenimiento que formadores e informadores de la sociedad, que menoscaban, en ocasiones, la percepción sobre la calidad de dichos contenidos (Farias y Roses 2009: 16). En este sentido y atendiendo a lo sucedido en los últimos años en el sector audiovisual español, destaca el fenómeno de la denominada "telebasura". Algo parecido, ocurre con la búsqueda de nuevos formatos.

Las nuevas tecnologías ofrecen un canal suculento para cualquiera: barato, rápido, cuya penetración se multiplica casi al minuto y reporta a los medios ciertos matices de prestigio y modernidad (Viñarás Abad, 2013). Sin embargo, las prisas y la presión a las que se está sometiendo al profesional del periodismo en la elaboración de los materiales provocan una bajada alarmante de la calidad informativa. Quizá fue esa la causa de un caso que ha sentado precedentes en las empresas del sector de la comunicación de nuestro país.

Según la profesora María José Pérez Serrano, entrevistada para este artículo, "la lucha por las audiencias es una realidad que en los últimos tiempos ha tomado tintes dramáticos". "Pero si algo hay seguro, por precedentes históricos, es que la crisis financiera pasará y se llevará con ella a algunos medios", afirma la investigadora de la UCM.

\section{A modo de conclusión.}

En estos momentos, al finalizar el año 2013, la economía española sigue siendo víctima de una crisis económica global. En el caso del mercado de la comunicación, puesto que estamos hablando de un negocio de marcado signo internacional, las consecuencias son muy duras y negativas y se ceban con especial interés en el caso de nuestro país.

Esta realidad trae consigo consecuencias en la gestión en las filiales españolas que se traducen en una mayor presión contable, disminución de ingresos y reducción de márgenes de beneficios de las empresas de comunicación. Si hubiera que sintetizar en pocas líneas los aspectos que más están condicionando el marco de actuación de las empresas de publicidad y que mayor peso e influencia están teniendo en la restructuración del mercado de agencias en España, cabría señalar la potente emergencia de las redes digitales y el declive y fragmentación de los medios convencionales, junto a nuevos usos sociales. 
1.- La inversión publicitaria en soportes y medios digitales es la única que nos permite esbozar un futuro de esperanza. Además, en términos de la propia comunicación publicitaria, habría que hablar de un escenario marcado por la confluencia entre la emergencia de nuevas formas de publicidad asociadas a la denominada web social y la telefonía móvil (smartphones) y el progresivo retroceso de la publicidad convencional.

2.- En el actual entorno o contexto de cambio casi constante o permanente, de momento, las empresas de publicidad no pueden volcarse únicamente en una sola fórmula. Aunque en general todos los profesionales de las agencias pueden llegar a estimar hoy en día que el futuro más inmediato será el de un escenario de equilibrio en el que coexistirán la publicidad convencional y la digital durante los próximos años, habrá que ir haciéndose a la idea de que las agencias de creatividad, marketing interactivo y consultorías de marca son el futuro a medio y largo plazo y no muy tarde acabará por imponerse la comunicación digital (García López \& Martínez Pastor, 2013).

3.- El análisis de las nuevas demandas del mercado nos hace darnos cuenta de una nueva realidad. Se busca cada vez más que las empresas de comunicación estén orientadas hacia una gestión más coherente e integrada de las marcas y su publicidad en una comunicación bidireccional con los clientes y usuarios, en conclusión, más cerca de sus públicos. La industria actual de las agencias poco tiene que ver con este retrato de un modelo de agencias de antaño que ya no existe.

Corren nuevos tiempos para el mercado de la comunicación en general y de la publicidad en particular. Vivimos en una nueva sociedad digital que demanda nuevas formas de comunicarse. Se han superado los paradigmas comunicativos tradicionales y estamos, como siempre, en una permanente operación de cambio, por lo que no podemos dejar de mirar hacia el futuro.

\section{Bibliografía y fuentes}

BENÍTEZ, J. J. \& CABRERA, S. (2010). "La capacidad de la Unión Europea para fomentar la recuperación económica". Boletín económico del ICE, núm. 2985, pp. 11-18.

CALDEVILLA DOMÍNGUEZ, D. (2013). Nuevas fórmulas de periodismo. Periodismo de proximidad 2.0. CIC: Cuadernos de información y comunicación. Núm. 18. Págs. 165-176

CALDEVILLA DOMÍNGUEZ, D. (2013). Efectos actuales de la sobreinformación y la infoxicación a través de las bitácoras y del proyecto I+D Avanza 'Radiofriends'. Revista de la SEECI. Núm. 30. Págs. 34-56.

FARIAS, P. Y ROSES, S. (2009). "La crisis acelera el cambio del negocio informativo". Estudios sobre el Mensaje Periodístico, núm. 15: pp.15-32. 
FERNÁNDEZ SANDE, M. (2013). La crisis de la prensa en España: análisis del caso Público, un diario nacido a contracorriente. Revista de Comunicación 'Vivat Academia'. № 122. Págs. 22-39.

GARCÍA LOPÉZ, J. \& MARTÍNEZ PASTOR, E. (2013). Investigación en publicidad digital en revistas científicas españolas. Revista El Profesional de la Información (EPI). Número de marzo-abril de 2013. Vol. 22. Págs. 173-180.

INFOADEX. Estudio Infoadex de la inversión publicitaria en España 2010 [Documento de Internet disponible en: http://www.infoadex.es/estudios/resumen2010. pdf]

INFOADEX. Estudio Infoadex de la inversión publicitaria en España 2012 [Documento de Internet disponible en: http://www.infoadex.es/estudios/resumen2012. pdf]

INSTITUTO NACIONAL DE ESTADÍSTICA (INE). 2013. Encuesta de Población Activa (EPA), primer trimestre de 2013 [Documento de Internet disponible en: http://www.ine.es]

NADAL, Á. (2008). "La crisis financiera de Estados Unidos". Boletín económico del ICE, núm. 2953: pp. 19-29.

PÉREZ SERRANO, $\mathrm{M}^{\mathrm{a}} \mathrm{J}$. (2010): “Análisis del valor de las empresas informativas", en Palabra Clave, vol. 13, 1, pp. 47-58 (Colombia). ISSN: 0122-8285.

PÉREZ SERRANO, $M^{\mathrm{a}} \mathrm{J}$. \& ROMERO CALMACHE, $\mathrm{M}^{\mathrm{a}}$. (2009): La gestión de las personas en el nuevo entorno empresarial de la comunicación. Vivat Academia. $\mathrm{n}^{\mathrm{o}}$ 109. Diciembre-Enero. 2009- 2010. Págs. 1-23.

ROMERO CALMACHE, $\mathrm{M}^{\mathrm{a}}$; PÉREZ SERRANO, $\mathrm{M}^{\mathrm{a}}$. J.; OROZ ARTIGAS, S. (2010): "Prospectiva de la crisis mediática en un contexto de crisis global", en Re-Presentaciones, 6, pp. 73-94 (Chile). ISSN: 0718-4263.

SOTELO GONZÁLEZ, J., (2011) "Si McLuhan levantara la cabeza... La computación en la nube y el nuevo paradigma socio cultural", Revista de Estudios de Juventud, $\mathrm{n}^{\circ}$ 92, Instituto de la Juventud (INJUVE), Madrid, pp. 111-121. ISSN: $0211-4364$

SOTELO GONZÁLEZ, J.; SALGADO SANTAMARÍA, C. (2011): "Que alguien nos diga cómo lo vamos a hacer. La difícil adaptación del periodismo tradicional en la era tecnológica", en VERÓN LASSA, J. J.; SABÉS TURMO, F., (coords.), La investigación en periodismo digital. Algunos trabajos desde el ámbito universitario (XII Congreso de Periodismo Digital), Asociación de Periodistas de Aragón, Ayuntamiento de Huesca, Huesca, pp. 181-195.

TAPIA FRADE, A., GÓMEZ NIETO, M ${ }^{\mathrm{a}}$ B., HERRANZ DE LA CASA, J. M ${ }^{\mathrm{a}}$ \& MATELLANES LAZO, Mónica (2010). Los estudiantes universiarios ante las redes sociales: cuestiones de uso y agrupación en estructuras elitistas o pluralistas. Revista de Comunicación 'Vivat Academia', Nª 113. Págs. 1-13.

VIÑARÁS ABAD, M. (2013). Estrategias de comunicación para generar confianza, en Revista Comunicación y Hombre, $\mathrm{N}^{\mathrm{0}}$ 9, Madrid, Editorial Universidad Francisco de Vitoria. 


\section{Notas}

Este trabajo no habría sido posible sin la colaboración de la profesora Dra. María José PÉREZ SERRANO, investigadora y docente del Departamento de Periodismo IV (Empresa Informativa) de la Facultad de Ciencias de la Información de la Universidad Complutense de Madrid (UCM).

\section{El autor}

Francisco Cabezuelo Lorenzo es Doctor Europeo en Tecnologías, Estructuras y Tratamientos de la Información (UCM). Cuenta con un Máster Oficial Universitario en Marketing y Comunicación Corporativa (USJ). Ha sido becario de postgrado de la Fundación "la Caixa" en Public Relations Management (McGill, Canadá) y de la Fundación Caja Madrid en The Queen's University of Belfast (Irlanda del Norte). Es Licenciado en Periodismo (UCM) y Licenciado en Publicidad y RRPP (UCJC). En la actualidad es profesor en la Facultad de Ciencias Sociales, Jurídicas y de la Comunicación del Campus de Segovia de la Universidad de Valladolid (España). Gracias a los programas académicos de cooperación Erasmus ha sido profesor visitante en universidades de Bélgica, Italia, Países Bajos y Reino Unido. 